\title{
The ReWiSed CARe Technique
}

\section{Simultaneous Treatment of Atherosclerotic Tandem Occlusions in Acute Ischemic Stroke}

\author{
Volker Maus ${ }^{1,2}$ (D) $\cdot$ Daniel Behme ${ }^{1} \cdot$ Christoph Maurer $^{3} \cdot$ Andrei Tropine $^{4} \cdot$ Stephanie Tritt ${ }^{4} \cdot$ Ansgar Berlis $^{3}$. \\ Marios-Nikos Psychogios ${ }^{1,5}$
}

\begin{abstract}
Background Endovascular treatment of acute cerebral large vessel occlusions and concomitant atherosclerotic lesions of the ipsilateral internal carotid artery (ICA) are a matter of debate. This article reports a multi-center experience using the retriever wire supported carotid artery revascularization (ReWiSed CARe) technique allowing simultaneous treatment of both lesions.

Methods The study retrospectively analyzed 23 patients with acute tandem occlusions (TO) who underwent ReWiSed CARe at 3 German centers. Clinical, including demographics and National Institutes of Health Stroke Scale (NIHSS) and procedural, including angiographic evaluation of recanalization, data were evaluated. Favorable clinical outcome was defined as modified Rankin Scale (mRS) score $\leq 2$ at 90 days.

Results The median age was 70 years (interquartile range [IQR] 65-80 years) and 70\% were male. The median baseline NIHSS was 15 (IQR 11-17). Out of 23 patients, 22 (96\%) had an intracranial occlusion of the anterior circulation. Successful stent retriever deployment with subsequent carotid artery treatment was feasible in all cases without displacement of the stent retriever during the procedure. Overall, successful reperfusion (modified Thrombolysis in Cerebral Infarction [mTICI] $\geq 2 \mathrm{~b}$ ) was achieved in 22/23 (96\%) patients with 10/23 (44\%) individuals completely reperfused (mTICI 3). The median groin puncture to stent retriever deployment was $29 \mathrm{~min}$ (IQR 23-46) and groin puncture to final revascularization was 63 min (IQR 56-78). The median NIHSS at discharge was 5 (IQR 3-12) with favorable clinical outcome at 90 days in 11 out of 20 patients $(55 \%)$.

Conclusion Endovascular treatment using ReWiSed CARe is safe, fast and efficient in TO patients with underlying atherosclerotic lesion of the extracranial ICA.
\end{abstract}

\footnotetext{
Volker Maus and Daniel Behme contributed equally to the manuscript.

Volker Maus

volker.maus@kk-bochum.de

1 Department of Neuroradiology, University Medical Center Göttingen, Göttingen, Germany
}

Institute of Diagnostic and Interventional Radiology, Neuroradiology and Nuclear Medicine, Knappschaftskrankenhaus Bochum, Ruhr University Bochum, In der Schornau 23-25, 44892 Bochum, Germany

3 Department of Neuroradiology, University Hospital Augsburg, Augsburg, Germany

4 Department of Radiology and Neuroradiology, Helios Hospital Wiesbaden, Wiesbaden, Germany

5 Department of Neuroradiology, Clinic of Radiology \& Nuclear Medicine, University Hospital Basel, Basel, Switzerland 


\section{Introduction}

A subgroup of acute ischemic stroke (AIS) patients suffer from a combination of an intracranial large vessel occlusion (LVO) and an extracranial internal carotid artery (ICA) high-grade stenosis or occlusion [1]. The prognosis of so-called tandem occlusions (TO) is poor when treated with intravenous thrombolysis (IVT) alone because of a reduced supply of the thrombolytic drug due to high amount of thrombus [2]. Although this subtype of stroke was either underrepresented or excluded in several randomized controlled trials published in 2015, intracranial mechanical thrombectomy (MT) has been shown to improve the clinical outcome in TO patients as demonstrated in subgroup analyses of ESCAPE and MRCLEAN [3, 4]. A recent meta-analysis established that nearly half of the TO patients treated with MT have a favorable clinical outcome [5]. Another important aspect is the treatment of the extracranial lesion as to date no standardized recommendation for the optimal strategy exists. Focusing only on the extracranial carotid artery lesion, several questions arise: first, the optimal timepoint of ICA recanalization in cases of high-grade stenosis as it is not clear if it should be done within the initial intervention when the intracranial lesion is recanalized or in a staged procedure days after MT [6]. Second, in terms of a one-stage treatment, further doubts exist if an antegrade approach should be executed comprising primary recanalization of the ICA with subsequent MT of the intracranial LVO or a retrograde strategy addressing first the intracranial occlusion followed by ICA recanalization [5, 7]. An alternative approach towards TO called retriever wire supported carotid artery revascularization (ReWiSed CARe) was recently described in a TO patient with an underlying ICA dissection [8]. This novel technique utilizes the wire of a stent retriever as the guiding wire for carotid artery stenting (CAS), which may restore antegrade flow through the released stent retriever during the procedural time of CAS.

The purpose of this multicenter study was to evaluate the feasibility, efficacy and safety of ReWiSed CARe in TO patients with underlying extracranial atherosclerotic carotid lesions.

\section{Methods}

\section{Study Design and Clinical Assessment}

In this multicenter study, data from AIS patients were retrospectively analyzed after treatment with the ReWiSed CARe technique due to an extracranial/intracranial TO in the time period between November 2016 and September 2018. Assessment of baseline characteristics, procedural data and clinical outcome achieved in the three participat-

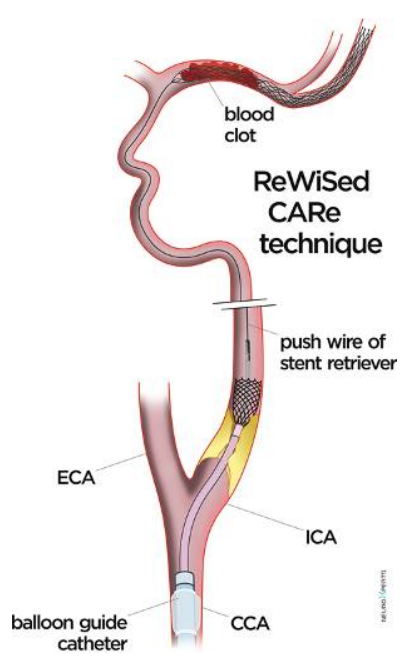

Fig. 1 Schematic illustration of ReWised CARe. The 8 French balloon guided catheter is placed at the distal common carotid artery. After the stent retriever is successfully deployed the push wire of the stent retriever is used as a microwire for the carotid artery stenting maneuver. Therefore, a carotid stent system is pushed to the level of the stenosis and implanted under continuous control of the stent retriever position. $C C A$ common carotid artery, ECA external carotis artery, ICA internal carotid artery

ing institutions (center A Göttingen, center B Augsburg, center C Wiesbaden) was carried out from prospectively collected databases. According to the guidelines of the respective local ethics committees, ethic approval was given when necessary for this anonymous retrospective study, which was conducted in accordance with the Declaration of Helsinki. Inclusion criteria were evidence of a proven high-grade stenosis ( $\geq 70 \%$ according to North American Symptomatic Carotid Endarterectomy Trial [NASCET] criteria) and occlusion of the extracranial ICA ipsilateral to an acute intracranial LVO based on computed tomography angiography (CTA) or digital subtraction angiography (DSA). All eligible patients received IVT based on the judgment of the attending neurologist according to the guidelines of the German Neurological Society. The patients were included independent of the administered antiplatelet regimen. There were no general limitations on baseline variables. The angiographic results were graded locally according to the modified thrombolysis in cerebral infarction (mTICI) score. All DSA images from center A were re-evaluated by an internal core team in accordance with the recommendations of the Cerebral Angiographic Revascularization Grading Collaborators [9], who was blinded to the clinical outcome and in an anonymous fashion to determine the extent of tissue reperfusion. The National Institute of Health Stroke Scale (NIHSS) and modified Rankin Scale (mRS) parameters were assessed by a stroke neurologist. All values are presented as median and interquartile range (IQR) or mean and standard deviation (SD) for continuous variables, and as frequency and percent for categorical variables.

\section{Endovascular Technique}

The ReWiSed CARe technique was described previously [8]. Briefly, in cases of TO an 8 French guide catheter (or optionally a balloon guide catheter) is advanced to the distal 
common carotid artery (CCA). A $160 \mathrm{~cm}$ microcatheter is navigated to the inferior trunk of the middle cerebral artery and a stent retriever, usually a $4 \times 20 \mathrm{~mm}$ pREset LITE (Phenox, Bochum, Germany), is deployed. After removal of the microcatheter the A C-arm is focused on the M1 segment and stent retriever while the B C-arm is used for depiction of the distal CCA and proximal ICA. The wire of the stent retriever (it is important to use stent retrievers with thin wires, e.g. $0.015 \mathrm{in}$. or $0,016 \mathrm{in}$.) is then used as a microwire for the CAS maneuver (Fig. 1). A carotid stent system is pushed to the level of the stenosis or occlusion and implanted under continuous control of the stent retriever position with subsequent use of a balloon angioplasty to optimize vessel wall reconstruction. Weight-adjusted heparin and aspirin are injected intravenously prior to the CAS maneuver. In the meantime, an aspiration catheter is prepared and navigated to the face of the clot. In cases where the guide catheter can be pushed distally to the carotid stent a SAVE (stent retriever assisted vacuum-locked extraction) thrombectomy maneuver is performed [10]. In cases where the guide catheter cannot be pushed distally to the carotid stent a Solumbra maneuver is performed [11].

\section{Results}

A total of 23 patients with acute TO were treated with ReWiSed CARe. Median age was 70 years (IQR 65-80) and $70 \%$ were male (Table 1). Out of 23 patients, $22(96 \%)$ had an intracranial occlusion of the anterior circulation: distal ICA in $11 / 23(48 \%)$, MCA M1 in 9/23 (39\%), and MCA M2 in 2/23 (9\%). One patient (4\%) suffered from basilar artery occlusion and a stenosis of the feeding vertebral artery. Median baseline NIHSS was 15 (IQR 11-17) and median Alberta Program Early CT Score (ASPECTS) on initial non-contrast computed tomography was 8 (IQR 8-9). In 78\% IVT was given prior to the intervention.

Performing the ReWiSed CARe technique including successful stent retriever deployment with subsequent carotid artery treatment was feasible in all cases. No dislocation of the distally placed stent retriever occurred during CAS. The median groin puncture to stent retriever deployment time was $29 \mathrm{~min}$ (IQR 23-46) and the groin puncture to final revascularization time was $63 \mathrm{~min}$ (IQR 56-78). Overall, successful reperfusion (mTICI $\geq 2$ b) was achieved in $22 / 23$ (96\%) patients with 10/23 (44\%) individuals completely reperfused (mTICI 3). First-pass complete reperfusion was done in 7/23 (30\%) patients. Mean number of stent retriever passes was $1.2 \pm 0.4$. In 20/23 (87\%) cases, the extracranial carotid artery lesion was treated with stent implantation and balloon angioplasty without the need of predilatation. For the remaining, no stent implantation but balloon angioplasty alone was executed: 2 patients aged 88 years showed
Table 1 Demographic, procedural and clinical parameters

\begin{tabular}{|c|c|}
\hline Number of patients, $n$ & 23 \\
\hline \multicolumn{2}{|l|}{ Baseline characteristics } \\
\hline Age (years), median (IQR) & $70(65-80)$ \\
\hline Male, $n(\%)$ & $16(70)$ \\
\hline Arterial hypertension, $n(\%)$ & $13(57)$ \\
\hline Atrial fibrillation, $n(\%)$ & $3(13)$ \\
\hline Diabetes mellitus, $n(\%)$ & $6(26)$ \\
\hline Chronic kidney disease, $n(\%)$ & $3(13)$ \\
\hline NIHSS baseline, median (IQR) & $15(11-17)$ \\
\hline IVT, $n(\%)$ & $18(78)$ \\
\hline ASPECTS baseline, median (IQR) & $8(8-9)$ \\
\hline \multicolumn{2}{|l|}{ Intracranial occlusion site } \\
\hline ICA, $n(\%)$ & $11(48)$ \\
\hline MCA, M1, $n(\%)$ & $9(39)$ \\
\hline $\mathrm{MCA}, \mathrm{M} 2, n(\%)$ & $2(9)$ \\
\hline $\mathrm{BA}, n(\%)$ & $1(4)$ \\
\hline \multicolumn{2}{|l|}{ Procedural data } \\
\hline General anesthesia, $n(\%)$ & $16(70)$ \\
\hline Onset-admission [min], median (IQR) & $162(83-209)$ \\
\hline Admission-groin puncture [min], median (IQR) & $45(27-58)$ \\
\hline $\begin{array}{l}\text { Groin puncture-SR deployment [min], median } \\
\text { (IQR) }\end{array}$ & $29(23-46)$ \\
\hline $\begin{array}{l}\text { Groin puncture-final recanalization [min], median } \\
\text { (IQR) }\end{array}$ & $63(56-78)$ \\
\hline First-pass complete reperfusion (mTICI 3), $n(\%)$ & $7(30)$ \\
\hline $\begin{array}{l}\text { First-pass successful reperfusion (mTICI } \geq 2 \mathrm{~b}), n \\
(\%)\end{array}$ & $19(83)$ \\
\hline Overall complete reperfusion (mTICI 3), $n(\%)$ & $10(44)$ \\
\hline Overall successful reperfusion (mTICI $\geq 2 b), n(\%)$ & $22(96)$ \\
\hline Number of SR passes, mean \pm SD & $1.2 \pm 0.4$ \\
\hline Emboli in new territories, $n(\%)$ & $1(4)$ \\
\hline Distal embolization, $n(\%)$ & $3(13)$ \\
\hline Subarachnoid hemorrhage, $n(\%)$ & $3(13)$ \\
\hline Intracranial parenchymal hemorrhage, $n(\%)$ & $1(4)$ \\
\hline Symptomatic intracranial hemorrhage, $n(\%)$ & - \\
\hline \multicolumn{2}{|l|}{ Clinical outcome } \\
\hline NIHSS 24h, median (IQR) & $5(7-17)$ \\
\hline NIHSS at discharge, median (IQR) & $5(3-12)$ \\
\hline $\mathrm{mRS} \leq 2$ at discharge, $n(\%)$ & $11 / 23(48)$ \\
\hline $\mathrm{mRS} \leq 2$ after 90 days, $n(\%)$ & $11 / 20(55)$ \\
\hline Mortality, periprocedural, $n(\%)$ & $3 / 20(15)$ \\
\hline Mortality after 90 days, $n(\%)$ & $4 / 20(20)$ \\
\hline ASPECTS $24 \mathrm{~h}$, median (IQR) & $7(6-8)$ \\
\hline
\end{tabular}

ASPECTS Alberta Stroke Program Early CT Score, $B A$ basilar artery, $I C A$ internal carotid artery, IQR interquartile range, IVT Intravenous thrombolysis, $M C A$ middle cerebral artery, $m R S$ modified Rankin Scale, $m$ TICI modified thrombolysis in cerebral infarction, NIHSS National Institute of Health Stroke Scale, SR stent retriever, $S D$ standard deviation 

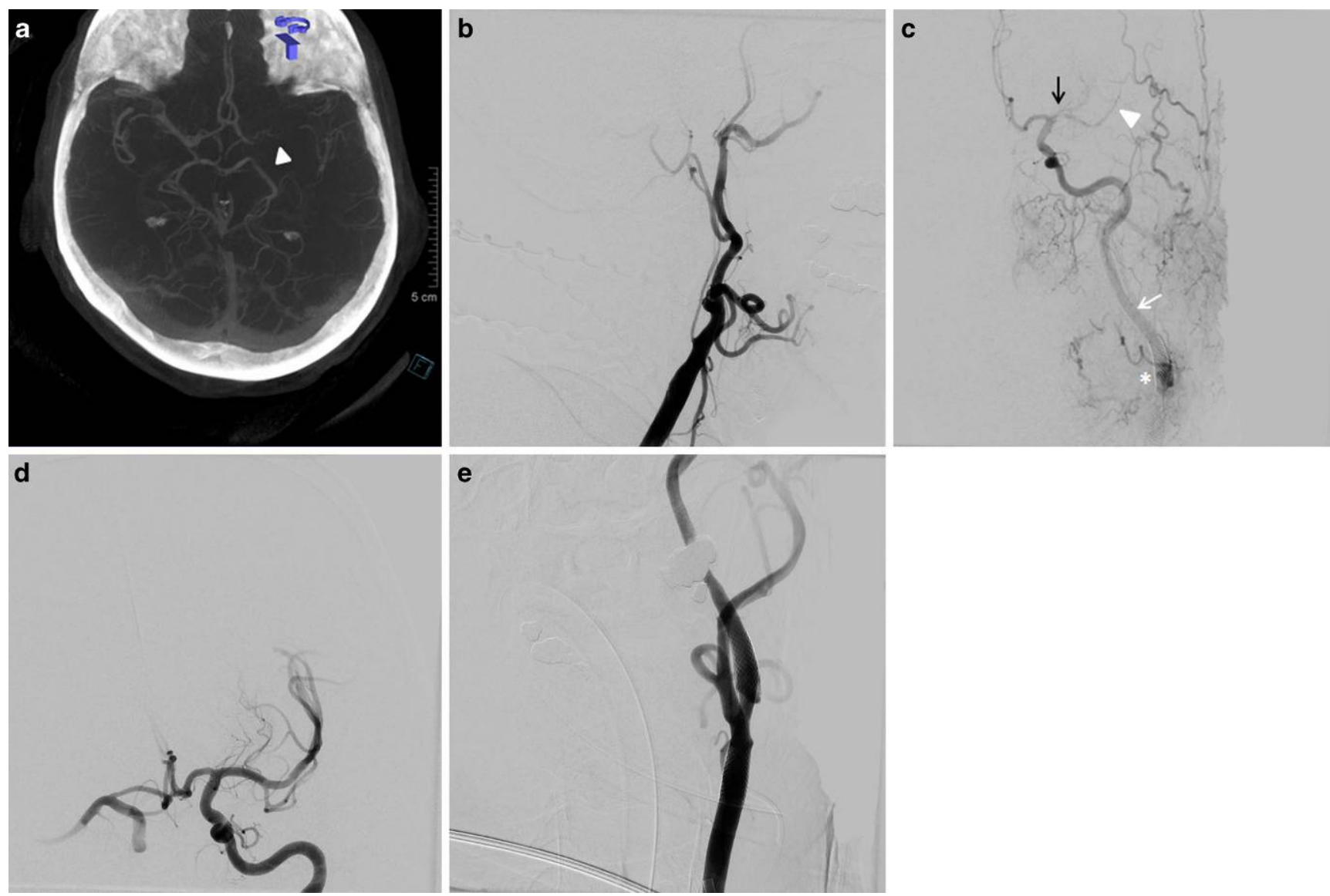

Fig. 2 a Baseline flat-detector computed tomography of a patient suffering from an occlusion of the left middle cerebral artery (MCA, white arrowhead). b Initial lateral angiogram of the common carotid artery depicting an occlusion of the internal carotid artery (ICA) ipsilateral to the intracranial lesion. c After the occlusion is passed with the microcatheter, a stent retriever is deployed in the MCA (black arrow) and antegrade flow to the affected territory is achieved early during the procedure (white arrowhead). The wire of the stent retriever (white arrow) is utilized for simultaneous deployment of the carotid stent (asterisk). d After retrieval of the stent retriever successful recanalization is achieved. e Final lateral angiogram of the extracranial carotid artery demonstrating good vessel wall reconstruction of the ICA

increased signs of ischemic damage with low ASPECTS (5 and 7, respectively) due to an extended time window in a drip-and-ship model with a time from symptom onset to groin puncture of $273 \mathrm{~min}$ and $264 \mathrm{~min}$, respectively; stent implantation and antiplatelet medication were avoided to reduce the potential risk of intracranial hemorrhage. Another patient with a basilar artery occlusion also underwent dilatation of the feeding vertebral artery.

In 3/23 (13\%) individuals, a distal embolization occurred: in one patient with an ICA-T occlusion, the MCA territory was reperfused completely after the first attempt; however, a proximal A2 embolization made a second pass necessary. In two patients suffering from an ICA-T and M1 occlusion, respectively, a distal embolization of the proximal M3 segment remained. Subarachnoid hemorrhage of limited extent occurred in 3/23 (13\%) patients. The rate of symptomatic intracranial hemorrhage was $0 \%$. An exemplary case is shown in Fig. 2.
Perioperative antiplatelet medication was only administered in patients who were treated with extracranial carotid stenting. Out of 20 patients, $17(85 \%)$ received loading with aspirin; additionally, in 2/17 (12\%) weight-adjusted abciximab and in $1 / 17(6 \%)$ clopidogrel was given after the procedure. Of the patients two $(10 \%)$ received only weight-adjusted abciximab and one (5\%) patient did not receive any loading medication due to pre-existing aspirin monotherapy. After intracranial hemorrhage was excluded, lifelong aspirin and additional clopidogrel administration were given, the latter varied from 6 weeks to 6 months. The median NIHSS at discharge was 5 (IQR 3-12) and favorable clinical outcome $(\mathrm{mRS} \leq 2)$ was achieved in $11 / 23$ (48\%). The follow-up data after 90 days were available for 20/23 (87\%) patients with $11(55 \%)$ having a favorable clinical outcome; 4/20 (20\%) individuals died. 


\section{Discussion}

The results of this study show that ReWiSed CARe is a technical feasible approach for the treatment of atherosclerotic TO with $100 \%$ successful distal stent retriever placement and subsequent carotid artery treatment utilizing the stent retriever wire (stent or balloon angioplasty alone). Most interestingly, no displacement of the distally placed stent retriever occurred during CAS confirming the concept of a fixed wire to be an advantage in TO treatment as demonstrated in a patients suffering from ICA dissection [8]. Thereby, the technique is not associated with higher rates of ENT (embolization to new territory) compared to what has been reported when an antegrade or retrograde approach has been utilized to treat TO. In this study, only one ENT occurred (4\%) which is comparable to what has been reported by a recently published multicenter study with $4 \%$ ENT for the retrograde and $7 \%$ for the antegrade treatment of TO [7]. Furthermore, in this series no patient suffered from a sICH and only three patients suffered from a limited SAH without clinical sequelae. Large retrospective studies and a metareview found symptomatic intracranial hemorrhage ( $\mathrm{sICH})$ rates to be as high as 7-9\% [12, 13]; however, the absence of a sICH in this study has to be interpreted with caution due to the small number of cases included and additionally there were cases without CAS which did not receive antithrombotic medication. The occurrence of SAH after the procedure might possibly be based on the use of the microwire as it was recently shown that the probability of intracranial vessel perforation is reduced when passing the clot with a wireless microcatheter [14].

With an mTICI $2 b / 3$ rate of $96 \%$, patients treated by the means of ReWiSed CARe had substantially better reperfusion results than what has been reported for standard treatment of TO. Behme et al. reported a rate of only $70 \%$ successful reperfused patients in a large multicenter study [12]. A meta-analysis published in 2016 incorporating 237 patients found the TICI $2 \mathrm{~b} / 3$ rate to be $81 \%$ on average and the subgroup analysis of the ESCAPE trial reported $69 \% \mathrm{TICI} 2 \mathrm{~b} / 3$ results $[4,13]$. In a more recent study, Maus et al. found $91 \%$ of patients who were treated in a retrograde fashion to be successfully reperfused whereas antegrade treatment resulted in $63 \%$ mTICI $2 \mathrm{~b} / 3$ results which was a significant difference [7]. In addition to the better angiographic results, the results of this study showed that carotid artery treatment utilizing the stent retriever wire is a very fast approach towards tandem lesions. Groin-to-recanalization time in this study was $63 \mathrm{~min}$, which is considerably shorter compared to $88 \mathrm{~min}$ reported by Behme et al. or 103/123 min reported by Maus et al. when antegrade or retrograde approaches were carried out respectively [7, 12]; however, within the subgroup analysis of the ESCAPE trial patients were treated after a median of $88 \mathrm{~min}$ (CT to recanalization time) [4]; notably, only $57 \%$ of the 30 patients included in the ESCAPE analysis actually received treatment of the carotid lesion. Both the very high rates of successfully reperfused patients and the short groin to reperfusion times in this study translated into a rate of $48 \%$ favorable outcome at discharge and an even higher rate of $55 \%$ at 90 days, which is consistent with what has been reported in the literature for the outcome after interventional treatment of AIS caused by TO with rates ranging from $28-60 \%[4,7,12,13]$. A recent case series demonstrated advantages of a simultaneous treatment of proximal ICA lesions with balloon angioplasty in a TO situation; however, only five patients were included and carotid stenting was avoided [15].

Another aspect of ReWiSed CARe is the long dwell time of the stent retriever during the procedure, which might result in a better angiographic outcome. The long deployment of the stent retriever allows prolonged clot integration as this is known as a predictive factor of recanalization success [16]. Kannath et al. reported an improved angiographic outcome after $8 \mathrm{~min}$ stent retriever dwell time with fewer attempts compared to 3 and $5 \mathrm{~min}$, respectively [17].

Several limitations apply to this study. First of all, the retrospective design and the small cohort with the attendant selection bias and second the missing control group. In addition, treatment of the carotid lesion was not homogeneous (stent/balloon angioplasty), which might have affected some of the outcome measures that might depend on the medical treatment (e.g. intracranial hemorrhage).

\section{Conclusion}

Endovascular treatment using ReWiSed CARe is safe, fast and efficient in TO patients with underlying atherosclerotic lesion of the extracranial ICA. This technique seems to be an alternative strategy for this stroke subtype as the advantage of an antegrade flow restoration through the deployed stent retriever during the time of CAS might enable early reperfusion of the affected territory.

Conflict of interest V. Maus, D. Behme, A. Tropine, S. Tritt and A. Berlis declare that they have no competing interests. C. Maurer has educational grant (Microvention, Stryker). M.-N. Psychogios received travel grants and (modest) fees from Stryker, Phenox, Penumbra, Acandis and Siemens.

\section{References}

1. Goyal M, Demchuk AM, Menon BK, Eesa M, Rempel JL, Thornton J, Roy D, Jovin TG, Willinsky RA, Sapkota BL, Dowlatshahi D, Frei DF, Kamal NR, Montanera WJ, Poppe AY, Ryckborst KJ, Silver FL, Shuaib A, Tampieri D, Williams D, Bang OY, Baxter BW, Burns PA, Choe H, Heo JH, Holmstedt CA, Jankowitz B, Kelly M, Linares G, Mandzia JL, Shankar J, Sohn SI, Swartz RH, Barber PA, 
Coutts SB, Smith EE, Morrish WF, Weill A, Subramaniam S, Mitha AP, Wong JH, Lowerison MW, Sajobi TT, Hill MD; ESCAPE Trial Investigators. Randomized assessment of rapid endovascular treatment of ischemic stroke. N Engl J Med. 2015;372:1019-30.

2. del Zoppo GJ, Poeck K, Pessin MS, Wolpert SM, Furlan AJ, Ferbert A, Alberts MJ, Zivin JA, Wechsler L, Busse O, Greenlee R Jr, Brass L, Mohr JP, Feldmann E, Hacke W, Kase CS, Biller J, Gress D, Otis SM. Recombinant tissue plasminogen activator in acute thrombotic and embolic stroke. Ann Neurol. 1992;32:78-86.

3. Berkhemer OA, Borst J, Kappelhof M, Yoo AJ, van den Berg LA, Fransen PSS, Beumer D, Schonewille WJ, Nederkoorn PJ, Wermer MJH, Marquering HA, Lingsma HF, Roos YBWEM, van Oostenbrugge RJ, Dippel DWJ, van Zwam WH, Majoie CBLM, Emmer BJ, van der Lugt A; MR CLEAN Investigators. Extracranial carotid disease and effect of intra-arterial treatment in patients with proximal anterior circulation stroke in MR CLEAN. Ann Intern Med. 2017;166:867-75.

4. Assis Z, Menon BK, Goyal M, Demchuk AM, Shankar J, Rempel JL, Roy D, Poppe AY, Yang V, Lum C, Dowlatshahi D, Thornton J, Choe H, Burns PA, Frei DF, Baxter BW, Hill MD; ESCAPE Trialists. Acute ischemic stroke with tandem lesions: Technical endovascular management and clinical outcomes from the ESCAPE trial. J Neurointerv Surg. 2018;10:429-33.

5. Wilson MP, Murad MH, Krings T, Pereira VM, O'Kelly C, Rempel J, Hilditch CA, Brinjikji W. Management of tandem occlusions in acute ischemic stroke-Intracranial versus extracranial first and extracranial stenting versus angioplasty alone: A systematic review and meta-analysis. J Neurointerv Surg. 2018;10:721-8.

6. Akpinar CK, Gürkaş E, Aytac E. Carotid angioplasty-assisted mechanical thrombectomy without urgent stenting may be a better option in acute tandem occlusions. Interv Neuroradiol. 2017;23: 405-11

7. Maus V, Borggrefe J, Behme D, Kabbasch C, Abdullayev N, Barnikol UB, Yeo LLL, Brouwer PA, Söderman M, Möhlenbruch M, Liebig T, Fink GR, Mpotsaris A. Order of treatment matters in ischemic stroke: Mechanical thrombectomy first, then carotid artery stenting for tandem lesions of the anterior circulation. Cerebrovasc Dis. 2018;46:59-65.

8. Behme D, Knauth M, Psychogios MN. Retriever wire supported carotid artery revascularization (ReWiSed CARe) in acute ischemic stroke with underlying tandem occlusion caused by an internal carotid artery dissection: Technical note. Interv Neuroradiol. 2017;23:289-92.

9. ZZaidat OO, Yoo AJ, Khatri P, Tomsick TA, von Kummer R, Saver JL, Marks MP, Prabhakaran S, Kallmes DF, Fitzsimmons BF, Mocco J, Wardlaw JM, Barnwell SL, Jovin TG, Linfante I, Siddiqui AH, Alexander MJ, Hirsch JA, Wintermark M, Albers G,
Woo HH, Heck DV, Lev M, Aviv R, Hacke W, Warach S, Broderick J, Derdeyn CP, Furlan A, Nogueira RG, Yavagal DR, Goyal M, Demchuk AM, Bendszus M, Liebeskind DS; Cerebral Angiographic Revascularization Grading (CARG) Collaborators; STIR Revascularization working group; STIR Thrombolysis in Cerebral Infarction (TICI) Task Force. Recommendations on angiographic revascularization grading standards for acute ischemic stroke: A consensus statement. Stroke. 2013;44:2650-63.

10. Maus V, Henkel S, Riabikin A, Riedel C, Behme D, Tsogkas I, Hesse AC, Abdullayev N, Jansen O, Wiesmann M, Mpotsaris A, Psychogios MN. The SAVE technique. Clin Neuroradiol. $2018 \mathrm{Jul}$ 19. doi: 10.1007/s00062-018-0702-4. [Epub ahead of print]

11. Humphries W, Hoit D, Doss VT, Elijovich L, Frei D, Loy D, Dooley G, Turk AS, Chaudry I, Turner R, Mocco J, Morone P, Fiorella D, Siddiqui A, Mokin M, Arthur AS. Distal aspiration with retrievable stent assisted thrombectomy for the treatment of acute ischemic stroke. J Neurointerv Surg. 2015;7:90-4.

12. Behme D, Mpotsaris A, Zeyen P, Psychogios MN, Kowoll A, Maurer CJ, Joachimski F, Liman J, Wasser K, Kabbasch C, Berlis A, Knauth M, Liebig T, Weber W. Emergency stenting of the extracranial internal carotid artery in combination with anterior circulation thrombectomy in acute ischemic stroke: A retrospective multicenter study. AJNR Am J Neuroradiol. 2015;36:2340-5.

13. Sivan-Hoffmann R, Gory B, Armoiry X, Goyal M, Riva R, Labeyrie PE, Lukaszewicz AC, Lehot JJ, Derex L, Turjman F. Stent-retriever thrombectomy for acute anterior ischemic stroke with tandem occlusion: A systematic review and meta-analysis. Eur Radiol. 2017;27:247-54.

14. Keulers A, Nikoubashman O, Mpotsaris A, Wilson SD, Wiesmann M. Preventing vessel perforations in endovascular thrombectomy: Feasibility and safety of passing the clot with a microcatheter without microwire: The wireless microcatheter technique. J Neurointerv Surg. 2018 Dec 7. doi: 10.1136/neurintsurg-2018-014267. [Epub ahead of print]

15. Sultan-Qurraie A, Witt T, de Havenon A, Ribo M, Zaidat OO. SEIMLESS: Simultaneous extracranial, intracranial management of (tandem) lessions in stroke. J Neurointerv Surg. 2019 Jan 23. doi: 10.1136/neurintsurg-2018-014403. [Epub ahead of print]

16. Yi HJ, Lee DH, Sung JH. Clinical usefulness of waiting after stent deployment in mechanical thrombectomy: Effect of the clot integration. World Neurosurg. 2018;119:e87-e93.

17. Kannath SK, Rajan JE, Sylaja PN, Sarma PS, Sukumaran S, Sreedharan SE, Kapilamoorthy TR. Dwell time of stentriever influences complete revascularization and first-pass TICI 3 revascularization in acute large vessel occlusive stroke. World Neurosurg. 2018;110:169-73. 